\title{
Finger Stiffness
}

\author{
Thijs C.H. Oosterhoff • Sjoerd P.F.T. Nota $・$ David Ring
}

Received: 4 May 2014 / Accepted: 20 July 2014 / Published online: 13 November 2014

(C) Society of the Hand \& Microsurgeons of India 2014

\begin{abstract}
Background Finger stiffness varies substantially in patients with hand and upper extremity illness and can be notably more than expected for a given pathophysiology. In prior studies, pain intensity and magnitude of disability consistently correlate with coping strategies such as catastrophic thinking and kinesiophobia, which can be characterized as overprotectiveness. In this retrospective study we address the primary research question whether patients with finger stiffness are more often overprotective when the primary pathology is outside the hand (e.g. distal radius fracture) than when it is located within the hand. Methods In an orthopaedic hand surgery department 160 patients diagnosed with more finger stiffness than expected for a given pathophysiology or time point of recovery between December 2006 and September 2012 were analyzed to compare the proportion of patients characterized as overprotective for differences by site of pathology: (1) inside the hand, (2) outside the hand, and (3) psychiatric etiology (e.g. clenched fist). Results Among 160 subjects with more finger stiffness than expected, 132 (82\%) were characterized as overprotective including 88 of 108 (81\%) with pathology in the hand, 39 of $44(89 \%)$ with pathology outside the hand, and 5 of 8 (63\%) with psychiatric etiology. These differences were not significant. Conclusions Overprotectiveness is common in patients with more finger stiffness than expected regardless the site and type of primary pathology. It seems worthwhile to recognize and treat maladaptive coping strategies early during recovery to limit impairment, symptoms, and disability.
\end{abstract}

T. C. Oosterhoff $(\bowtie) \cdot$ S. P. Nota $\cdot$ D. Ring

Orthopaedic Hand Service, Yawkey Center, Suite 2100,

Massachusetts General Hospital, 55 Fruit Street, Boston,

MA 02114, USA

e-mail: dring@partners.org
Keywords Finger stiffness · Pain catastrophizing · Kinesiophobia · Pain · Disability · Overprotectiveness

\section{Introduction}

Pain intensity and magnitude of disability can be notably more or less than expected based on identifiable pathophysiology in the hand and upper extremity [1]. For example, some patients recovering from fracture of the distal radius develop finger stiffness while others do not and this seems only partly related to the complexity of the fracture and associated soft-tissue injury [2].

Pain intensity and magnitude of disability consistently correlate with overprotectiveness (catastrophic thinking [3-12]; kinesiophobia [3, 13, 14, 8, 9]), low self-efficacy [10] (a sense that one can achieve one's goals); and symptoms of depression $[3,15,11,12]$ across the spectrum of hand and upper extremity illness. We had the impression that finger stiffness in the absence of pathophysiology such as osteoarthritis or burn contractures (i.e. more finger stiffness than expected for a given disease or point of recovery) was strongly related to an overprotective mindset. In other words, the patients most prone to stiffness are the ones that find it more counterintuitive that uncomfortable stretching exercises will regain motion and restore function to the hand.

We reviewed patients diagnosed with more stiffness than expected for a given pathophysiology or time point of recovery to address the primary research question whether patients with finger stiffness are more often overprotective when the primary pathology is outside the hand (e.g. distal radius fracture) than when it is located within the hand. We also addressed several secondary study questions addressing the differences in protectiveness based on traumatic vs. nontraumatic primary pathology, operative vs. non-operatively treated patients and prolonged (greater than 4 weeks) 
immobilization. The portions of comorbid major depression were looked at as well to assess differences based on site of primary pathology.

\section{Materials and Methods}

This retrospective single-surgeon cohort study was approved by our Institutional Review Board. We started with 190 patients that received the ICD-9 code 718.44 (Contracture of joint, hand) from a billing database that covers the period between December 2006 and September 2012. The ability to identify patients with more stiffness than expected was facilitated by the fact that this surgeon infrequently used this code for expected stiffness due to arthritis, Dupuytren disease, tendon injury, complex fractures, etc.

Four patients younger than 18 years were excluded from the study. Among the remaining 186 patients, 160 had more stiffness than expected, 3 were miscoded (i.e., they did not have finger stiffness), and 23 had a discrete non-capsular pathophysiology accounting for most of the stiffness ( 9 contractures due to nerve palsy or spasticity, 5 burn contractures, 3 Dupuytren contractures [not postoperative stiffness], 2 tendon ruptures, 2 compartment syndromes, 1 severe eczema, and 1 scleroderma).

Among the 160 patients with more stiffness than expected the following patient characteristics were obtained from the hospital electronic medical record (including information from all caregivers) in addition to demographic and injury/ disease characteristics: (1) a diagnosis of major depressive disorder; (2) overprotectiveness (which this surgeon consistently notes in the record when it is present); (3) prolonged (greater than 4 weeks) immobilization. Overprotectiveness is documented when a patient avoids or limits use of the hand and stretching exercises because they have a sense that they are causing harm in spite of consistent and repeated reassurance from both the surgeon and the hand therapist that they are a healthy and important part of recovery.

Three main groups were formed based on the location of the primary diagnosis, to be used for analysis. These three groups were comprised respectively of diagnoses located in the hand, those outside the hand, and patients with psychiatric disorders causing stiffness (e.g. clenched fist syndrome). Within the hand and non-hand groups we separated trauma from non-trauma and operative from non-operative treated patients, resulting in a total of 9 subgroups. (Table 2).

\section{Statistical Analysis}

Group and subgroups frequencies and percentage were determined for the following variables: age, sex, overprotectiveness, a diagnosis of major depression, an initial visit more 3 months after onset/injury, and prolonged immobilization.
Pearson Chi-square analyses were performed to look for differences in overprotectiveness and depression between the 3 main groups (hand, non-hand, psychiatric).

\section{Results}

Descriptive Analysis

The mean age of the 160 qualifying patients was 51 years (SD $=17$ years; range $20-95$ years) and $82(51 \%)$ were women. Forty-five patients $(28 \%)$ had a diagnosis of major depression in the medical record and the behavior of 132 patients $(83 \%)$ was considered to be overprotective. In the trauma subgroups 52 of $120(43 \%)$ patients had an interval of at least 3 months between the initial injury and the visit to the hand surgeon, 17 patients of $120(14 \%)$ had worn a cast or immobilizing splint for more than 4 weeks. (Table 1).

The most common diagnoses in the hand trauma pathology group were PIP sprains/dislocations and phalanx fractures. Finger stiffness from non-traumatic hand pathology was usually post-operative. The traumatic, non-hand stiffness was related to upper arm fractures, most commonly a fracture of the distal radius. The non-traumatic, non-hand stiffness was usually related to a peripheral mononeuropathy such as carpal tunnel syndrome. (Table 2).

Table 1 Patient details

\begin{tabular}{lll}
\hline & $\mathrm{n}=160$ & \\
\hline Parameter & Mean & Range \\
Age (y) & 51 & $20-95$ \\
& Number & $\%$ \\
Sex & & \\
$\quad$ Male & 78 & 49 \\
Female & 82 & 51 \\
Patient characteristics & & \\
Diagnosis of depression & & \\
Yes & 45 & 28 \\
No & 115 & 72 \\
Overprotective & & 83 \\
Yes & 132 & 18 \\
$\quad$ No & 28 & \\
Illness characteristics & & \\
$>3$ months between injury and presenting to hand surgeon & \\
Yes & 56 & 35 \\
No & 104 & 65 \\
Y weeks cast or splint wear & & 11 \\
Yes & 173 & \\
\hline
\end{tabular}


Table 2 Diagnoses

\begin{tabular}{|c|c|c|c|c|c|c|c|c|c|}
\hline & $n=1$ & & & & & & & & \\
\hline & $\mathrm{n}$ & $\%$ & Age & Gender & & Overprotective & Depressed & & $>4$ weeks cast \\
\hline & & & Mean & $\sigma^{\pi}$ & q & n $(\%)$ & $\mathrm{n}(\%)$ & $\mathrm{n}(\%)$ & n $(\%)$ \\
\hline Hand located & 108 & 68 & 49 & $59(54 \%)$ & $50(46 \%)$ & $88(81 \%)$ & $27(25 \%)$ & $45(42 \%)$ & $6(5.6 \%)$ \\
\hline Trauma nonoperative & 61 & 38 & 48 & $36(59 \%)$ & $25(41 \%)$ & $47(77 \%)$ & $12(20 \%)$ & $30(49 \%)$ & $4(6.6 \%)$ \\
\hline PIP sprain/dislocation & 34 & 21 & & & & & & & \\
\hline Phalanx fracture & 12 & 7,5 & & & & & & & \\
\hline Finger laceration(s) & 9 & 5,6 & & & & & & & \\
\hline Metacarpal fracture & 4 & 2,5 & & & & & & & \\
\hline Hand laceration(s) & 1 & 0,6 & & & & & & & \\
\hline Ha.nd contusion & 1 & 0,6 & & & & & & & \\
\hline Trauma postoperative & 24 & 15 & 44 & $15(60 \%)$ & $10(40 \%)$ & $19(79 \%)$ & $5(21 \%)$ & $12(50 \%)$ & $1(4.2 \%)$ \\
\hline PIP Sprain/Dislocation & 1 & 0,6 & & & & & & & \\
\hline Phalanx fracture & 14 & 8,8 & & & & & & & \\
\hline Tendon laceration & 5 & 3,1 & & & & & & & \\
\hline Extensor tendon repair & 3 & 1,9 & & & & & & & \\
\hline FDP avulsion & 1 & 0,6 & & & & & & & \\
\hline FDP repairs of LF, RF and SF & 1 & 0,6 & & & & & & & \\
\hline Metacarpal fracture & 3 & 1,9 & & & & & & & \\
\hline Hand lacerations & 1 & 0,6 & & & & & & & \\
\hline Non trauma nonoperative & 7 & 4,4 & 60 & $2(29 \%)$ & $5(71 \%)$ & $6(86 \%)$ & $4(57 \%)$ & $0(0 \%)$ & $0(0 \%)$ \\
\hline Trigger finger(s) & 3 & 1,9 & & & & & & & \\
\hline Nonspecific Pain & 4 & 2,5 & & & & & & & \\
\hline Non trauma postoperative & 16 & 10 & 60 & $6(38 \%)$ & $10(63 \%)$ & $16(100 \%)$ & $6(38 \%)$ & $3(19 \%)$ & $1(6.3 \%)$ \\
\hline Dupuytren's contracture & 5 & 3,1 & & & & & & & \\
\hline Trigger finger(s) & 4 & 2,5 & & & & & & & \\
\hline Infection & 4 & 2,5 & & & & & & & \\
\hline Animal bite & 2 & 1,3 & & & & & & & \\
\hline Post operative & 1 & 0,6 & & & & & & & \\
\hline Septic wrist & 1 & 0,6 & & & & & & & \\
\hline $\begin{array}{l}\text { Trapezometacarpal OA, } \\
\text { s/p arthroplasty }\end{array}$ & 1 & 0,6 & & & & & & & \\
\hline Enchondroma & 1 & 0,6 & & & & & & & \\
\hline Ganglion Cyst & 1 & 0,6 & & & & & & & \\
\hline Non hand located & 44 & 28 & 61 & $17(40 \%)$ & $26(60 \%)$ & $39(89 \%)$ & $12(27 \%)$ & $10(23 \%)$ & $11(25 \%)$ \\
\hline Trauma nonoperative & 14 & 8,8 & 65 & $4(29 \%)$ & $10(71 \%)$ & $14(100 \%)$ & $1(7 \%)$ & $6(43 \%)$ & $6(43 \%)$ \\
\hline Distal radius/ulna fracture & 9 & 5,6 & & & & & & & \\
\hline Fracture proximal to distal radius & 3 & 1,9 & & & & & & & \\
\hline Elbow dislocation & 1 & 0,6 & & & & & & & \\
\hline Scaphoid fracture & 1 & 0,6 & & & & & & & \\
\hline Trauma postoperative & 21 & 13 & 57 & $8(40 \%)$ & $12(60 \%)$ & $17(81 \%)$ & $6(29 \%)$ & $4(19 \%)$ & $5(24 \%)$ \\
\hline Distal radius/ulna fracture & 13 & 8,1 & & & & & & & \\
\hline Fracture proximal to distal radius & 8 & 5,0 & & & & & & & \\
\hline Non trauma nonoperative & 7 & 4,4 & 64 & $4(57 \%)$ & $3(43 \%)$ & $7(100 \%)$ & $3(43 \%)$ & $0(0 \%)$ & $0(0 \%)$ \\
\hline $\begin{array}{l}\text { Median/ulnar neuropathy } \\
\text { (CubTS, CTS, nonlocalizing) }\end{array}$ & 7 & 4,4 & & & & & & & \\
\hline Non trauma postoperative & 2 & 1,3 & 55 & $1(50 \%)$ & $1(50 \%)$ & $1(50 \%)$ & $2(100 \%)$ & $0(0 \%)$ & $0(0 \%)$ \\
\hline $\begin{array}{l}\text { Median/ulnar neuropathy } \\
\text { (CTS, nonlocalizing) }\end{array}$ & 2 & 1,3 & & & & & & & \\
\hline Psychiatric Disorders & 8 & 5,0 & 65 & $2(25 \%)$ & $6(75 \%)$ & $5(63 \%)$ & $6(75 \%)$ & $1(13 \%)$ & $0(0 \%)$ \\
\hline Clenched fist syndrome & 7 & 4,4 & & & & & & & \\
\hline Secretan disorder & 1 & 0,6 & & & & & & & \\
\hline
\end{tabular}


Bivariate Analysis

There was no significant difference in overprotectiveness between patients with primary diagnoses located in the hand, primary diagnoses located outside the hand, and patients with psychiatric disorders $(\mathrm{p}=.098)$. There was a significant difference in comorbid major depression $(p=.010)$ between the main 3 groups attributable to patients with a psychiatric etiology for their hand stiffness ( 7 clenched fist syndromes and 1 Secretan disorder). There was no significant difference in comorbid major depression $(p=.78)$ between hand and nonhand primary pathology.

\section{Discussion}

Most patients with unexpected finger stiffness due to capsular contracture are overprotective, no matter the location or primary pathology. The prevalence of comorbid major depression is higher with $28 \%$ (95\% CI, 22-36) than the average of $6.6 \%(95 \% \mathrm{CI}, 5.9-7.3)$ for the population in the United States [16].

All research has limitations. First, the assessments of "more stiffness than expected" and "overprotective" are subjective and may be unreliable. On the other hand, we feel this is unlikely based on our knowledge of the scientific literature and discourse with other surgeons both formal and informal. The fact that the cohort consists of a single surgeon's patients is a strength in that we know that protectiveness is always assessed and documented, but it may limit the generalizability of the study if the patients in this surgeon's practice are different from the average patient with more finger stiffness than expected. In a retrospective study we have no measure of mood or mindset and must rely on the record documenting comorbid major depression. It is likely that many patients with this diagnosis did not have it documented in the record. In spite of these shortcomings we feel that this work provides a basis for future analysis of unexpected finger stiffness: stiffness that cannot be related to pathophysiology such as arthritis or injury. This study also supports the hypothesis that unexpected finger stiffness relates more to mindset and mood than to type and location of pathophysiology, although more study is needed.

Several studies document the independent negative effects of psychological traits forming overprotectiveness on pain and disability. For instance, decreases in kinesiophobia and pain catastrophizing during rehabilitation correlate with more improvement in pain and function during recovery from reconstruction of the anterior cruciate ligament [17]. In another study patients with greater catastrophic thinking exhibited less willingness to adopt a self-management approach to cope with pain [18].
Based on the data to date-including this study-it seems worthwhile to assess for maladaptive coping strategies and address them during the recovery process. There are both verbal and nonverbal clues that patients are feeling so protective that they may hinder their recovery. [19] There are also validated measures of hyper protectiveness including the Pain Catastrophizing Scale, the Pain Self-Efficacy Questionnaire, and PROMIS Pain Interference.[20] An approach to hand illness that accounts for the role of stress and coping strategies might limit disproportionate pain, disability, and recovery time compared to biomedical care alone (e.g. surgery, medication, splints, and exercises). Studies that address factors associated with unexpected finger stiffness prospective are merited and should address the psychological aspects of recovery.

Acknowledgements None

\section{References}

1. Ring D, Barth R, Barsky A (2010) Evidence-based medicine: disproportionate pain and disability. J Hand Surg Am 35 (8):1345-1347

2. Souer JS, Buijze G, Ring D (2011) A prospective randomized controlled trial comparing occupational therapy with independent exercises after volar plate fixation of a fracture of the distal part of the radius. J Bone Joint Surg Am 93(19):1761-1766. doi:10.2106/JBJS. J.01452

3. Das De S, Vranceanu AM, Ring DC (2013) Contribution of kinesophobia and catastrophic thinking to upper-extremity-specific disability. J Bone Joint Surg Am 95 (1):76-81

4. George SZ, Calley D, Valencia C, Beneciuk JM (2011) Clinical Investigation of Pain-related Fear and Pain Catastrophizing for Patients With Low Back Pain. Clin J Pain 27(2):108-115. doi:10. 1097/AJP.0b013e3181f21414

5. Scott W, Wideman TH, Sullivan MJ (2013) Clinically Meaningful Scores on Pain Catastrophizing Before and After Multidisciplinary Rehabilitation: A Prospective Study of Individuals With Subacute Pain After Whiplash Injury. Clin J Pain. doi:10.1097/AJP. 0b013e31828eee6c

6. Severeijns R, Vlaeyen JW, van den Hout MA, Weber WE (2001) Pain catastrophizing predicts pain intensity, disability, and psychological distress independent of the level of physical impairment. Clin J Pain 17(2): 165-172

7. Theunissen M, Peters ML, Bruce J, Gramke HF, Marcus MA (2012) Preoperative anxiety and catastrophizing: a systematic review and meta-analysis of the association with chronic postsurgical pain. Clin J Pain 28(9):819-841. doi:10.1097/AJP.0b013e31824549d6

8. Picavet HS, Vlaeyen JW, Schouten JS (2002) Pain catastrophizing and kinesiophobia: predictors of chronic low back pain. Am J Epidemiol 156(11):1028-1034

9. Peters ML, Vlaeyen JW, Weber WE (2005) The joint contribution of physical pathology, pain-related fear and catastrophizing to chronic back pain disability. Pain 113 (1-2):45-50

10. Pulvers K, Hood A (2013) The role of positive traits and pain catastrophizing in pain perception. Curr Pain Headache Rep 17(5): 330. doi:10.1007/s11916-013-0330-2

11. Bergbom S, Boersma K, Overmeer T, Linton SJ (2011) Relationship among pain catastrophizing, depressed mood, and outcomes across physical therapy treatments. Phys Ther 91 (5):754-764 
12. Linton SJ, Nicholas MK, MacDonald S, Boersma K, Bergbom S, Maher C, Refshauge K (2011) The role of depression and catastrophizing in musculoskeletal pain. Eur J Pain 15 (4): 416-422

13. Lentz TA, Sutton Z, Greenberg S, Bishop MD (2010) Pain-related fear contributes to self-reported disability in patients with foot and ankle pathology. Arch Phys Med Rehabil 91 (4):557-561

14. Lentz TA, Tillman SM, Indelicato PA, Moser MW, George SZ, Chmielewski TL (2009) Factors associated with function after anterior cruciate ligament reconstruction. Sports Health 1 (1):47-53. doi:10.1177/1941738108326700 10.1177_1941738108326700[pii]

15. Ring D, Kadzielski J, Fabian L, Zurakowski D, Malhotra LR, Jupiter JB (2006) Self-reported upper extremity health status correlates with depression. J Bone Joint Surg Am 88(9):1983-1988. doi:10.2106/ JBJS.E.00932

16. Kessler RC, Berglund P, Demler O, Jin R, Koretz D, Merikangas KR, Rush AJ, Walters EE, Wang PS (2003) The epidemiology of major depressive disorder: results from the National Comorbidity Survey Replication (NCS-R). JAMA 289(23):3095-3105. doi:10.1001/ jama.289.23.3095
17. Chmielewski TL, Zeppieri G, Jr., Lentz TA, Tillman SM, Moser MW, Indelicato PA, George SZ (2011) Longitudinal changes in psychosocial factors and their association with knee pain and function after anterior cruciate ligament reconstruction. Phys Ther 91 (9):1355-1366

18. Guite JW, Kim S, Chen CP, Sherker JL, Sherry DD, Rose JB, Hwang WT (2013) Pain Beliefs and Readiness to Change Among Adolescents With Chronic Musculoskeletal Pain and Their Parents Before an Initial Pain Clinic Evaluation. Clin J Pain. doi:10.1097/ AJP.0b013e31828518e9

19. Bot AG, Vranceanu AM, Herndon JH, Ring DC. (2012) Correspondence of patient word choice with psychologic factors in patients with upper extremity illness. Clin Orthop Relat Res. 2012 Nov;470(11):3180-6. doi:10.1007/s11999-012-2436-y Epub 2012 Jun 16. PubMed PMID: 22707072; PubMed Central PMCID: PMC3462883

20. Menendez ME, Bot AG, Hageman MG, Neuhaus V, Mudgal CS, Ring D. (2013) Computerized adaptive testing of psychological factors: relation to upper-extremity disability. J Bone Joint Surg Am. 2013 Oct 16;95(20):e149. doi:10.2106/JBJS.L.01614. PubMedPMID:24132364 